\title{
High-Dose Immunoglobulin G Therapy for Fulminant Myocarditis
}

\author{
Yutaka Takeda, MD; Satoshi Yasuda, MD; Shunichi Miyazaki, MD; \\ Satoshi Daikoku, MD; Satoshi Nakatani, MD; Hiroshi Nonogi, MD
}

\begin{abstract}
The cardiac function of an adult patient with fulminant myocarditis requiring a mechanical circulatory support was improved, along with the suppressed release of inflammatory mediators, after intravenous infusion with high-dose immunoglobulin. This therapy may have immunomodulatory effects and serve as a potential adjunctive therapy for patients with fulminant myocarditis. (Jpn Circ J 1998; 62: 871-872)
\end{abstract}

Key Words: Cytokines; Immune system, Immunoglobulin; Myocarditis; Nitric oxide

$\mathbf{H}$ igh-dose intravenous immunoglobulin $\mathrm{G}$ (IgG) has been emerging as a promising therapy for children with acute myocarditis! It potentially induces rapid resolution of left ventricular dysfunction in patients, and reduces mortality in experimental animal models? However, the mechanisms are not fully understood. In infectious diseases such as myocarditis, the release of endogenous inflammatory mediators influences cardiac function and may play an important pathophysiological role 3,4 We report an adult patient with fulminant myocarditis requiring mechanical circulatory support, in whom cardiac function was improved along with the suppressed release of inflammatory mediators after highdose intravenous $\operatorname{IgG}$ therapy.

\section{Case Report}

A 22-year-old woman was admitted with acute myocarditis, developing cardiogenic shock with a cardiac index of $1.2 \mathrm{~L} \mathrm{~min}^{-1} \mathrm{~m}^{-2}$. Echocardiography revealed generalized severe hypokinesis of the left ventricle with chamber enlargement. Because the conventional medical treatment was ineffective, percutaneous cardiopulmonary support (PCPS) was introduced to maintain blood pressure. Two days after PCPS, when cardiac function was further depressed (decreased \% fractional shortening and ejection time on echocardiography), the patient was treated with high-dose intravenous human polyclonal IgG infusion (2 $\mathrm{g} / \mathrm{kg}$ over $48 \mathrm{~h}$; Polyglobin®-N, Bayer)!,5 After IgG therapy, the release of cytokines (interferon- $y$, interleukin- $1 \beta$ and 6) and nitric oxide (its metabolites and second messenger) was suppressed without any adverse effects (Fig 1). Simultaneously, the levels of soluble intercellular adhesion molecule-1 decreased significantly. In the next few days, cardiac function had improved sufficiently enough to surgically remove the PCPS and to discontinue intravenous

(Received July 9, 1998; revised manuscript received August 10, 1998; accepted August 18, 1998)

Division of Cardiology, Department of Internal Medicine, National Cardiovascular Center, Suita, Osaka, Japan

Mailing address: Satoshi Yasuda, MD, Division of Cardiology, Department of Internal Medicine, National Cardiovascular Center, 57-1 Fujishiro-dai, Suita, Osaka 565, Japan. E-mail: syasuda@hsp. ncvc.go.jp catecholamines. The 2-month follow-up cardiac catheterization demonstrated no asynergy except for the posterolateral wall in the left ventricle, with recovery of pump function to $4.5 \mathrm{~L} \mathrm{~min}^{-1} \mathrm{~m}^{-2}$ of cardiac index and $50 \%$ of left ventricular ejection fraction. Epstein-Barr virus (EBV) infection was confirmed by polymerase chain reaction using the tissue obtained from endomyocardial biopsy. There were only borderline changes in lymphocytic infiltration in the cardiac tissue.

\section{Discussion}

In acute myocarditis, the immune responses triggered by the virus are responsible for the subsequent myocardial impairment? In the inflammatory process, macrophages and lymphocytes are activated to produce cytokines, the expression of which persists in the heart tissue for weeks in animal models of viral myocarditis6 Several cytokines, and their induction of nitric oxide, cause contractile depression and attenuate the positive inotropic response to $\beta$-adrenergic agonists, ${ }^{3,4}$ The expression of cell adhesion molecules is also enhanced by cytokines, which enables recruitment and further activation of immune cells, inducing myocardial injury. Therefore, cytokines play a key role in the pathogenesis of viral myocarditis. Studies in vitro demonstrate that $\operatorname{IgG}$ contains neutralizing antibodies to various cytokines? In addition, IgG directly downregulates cytokine production by blocking Fc receptors? These in vitro findings indicate that cytokines could be a potential therapeutic target of $\operatorname{IgG}$ for the modulation of the inflammatory cascade in viral myocarditis. We found overall suppression of cytokine release after IgG therapy, which may attenuate the vicious cycle of the immune response, contributing in part to the improvement of cardiac function in the present case. It is important to prove a cause and effect relationship regarding cardiac improvement with high-dose IgG therapy in a future study.

Viral infection has been suggested as a possible precursor of dilated cardiomyopathy? The immunomodulatory therapy with high-dose intravenous IgG may prevent the viral-mediated cardiac remodeling process proceeding to cardiomyopathy, which has recently been proven in patients. Although cardiac function in the present case was remarkably restored in 2 months, further follow-up is 


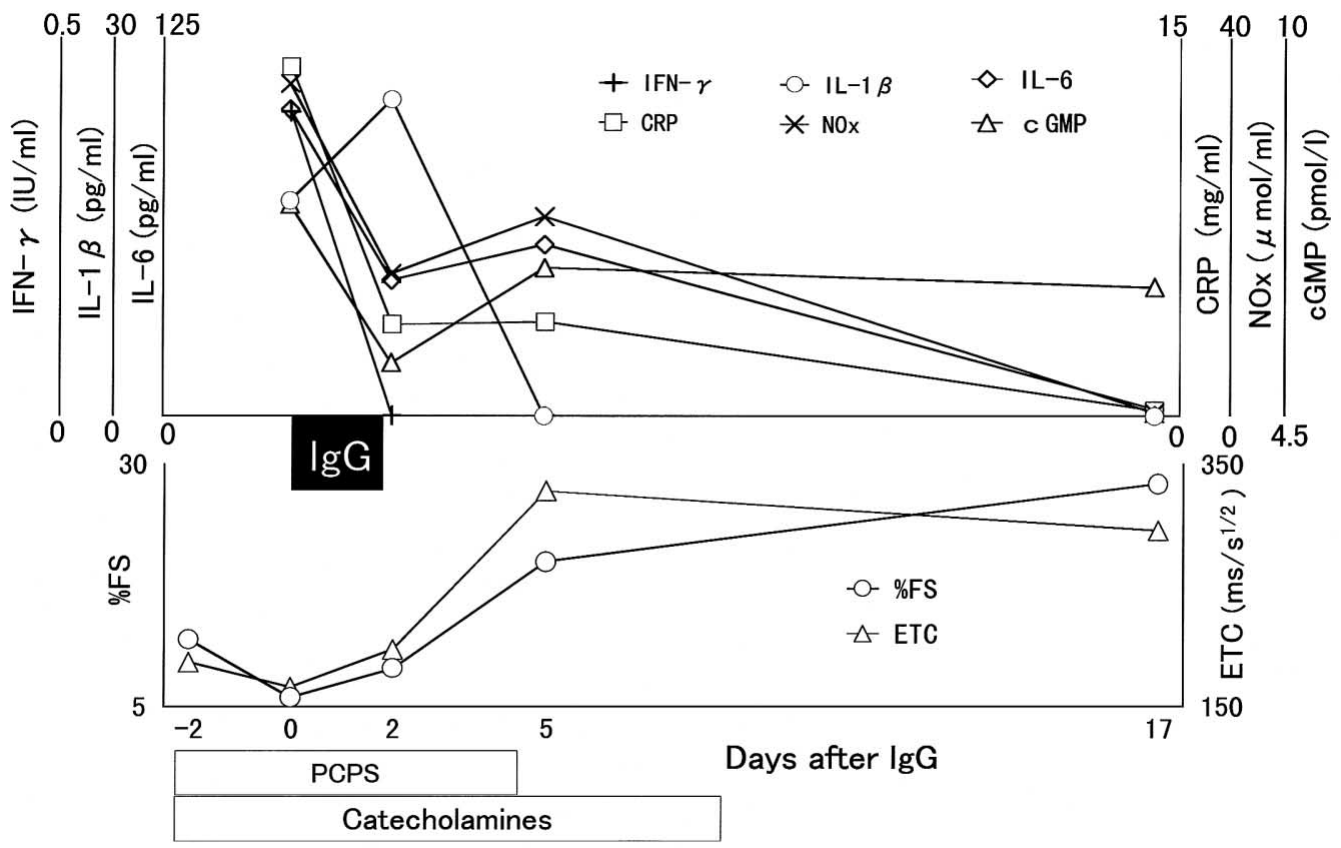

Fig 1. Changes in inflammatory mediators and cardiac function (evaluated by echocardiography). IFN $\gamma$, interferon- ; IL-1 , interleukin-1 ; IL-6, interleukin-6; CRP, C-reactive protein; NOx, nitrites and nitrates; cGMP, guanosine 3', 5'cyclic monophosphate; \%FS, \% fractional shortening; ETC, corrected ejection time (ejection time divided by the square root of RR interval); PCPS, percutaneous cardiopulmonary support; IgG, immunoglobulin G. The levels of tumor necrosis factora were undetectable.

needed to confirm persistent beneficial effects of IgG therapy.

In conclusion, the present findings suggest that highdose IgG may have immunomodulatory effects and serve as a potential adjunctive therapy for patients with viral myocarditis.

\section{References}

1. Drucker NA, Colan SD, Lewis AB, Beiser AS, Wessel DL, Takahashi M, et al:y-Globulin treatment of acute myocarditis in the pediatric population. Circulation 1994; 89: 252-257

2. Takada H, Kishimoto C, Hiraoka Y: Therapy with immunoglobulin suppresses myocarditis in a murine coxsackievirus B3 model: Antiviral and anti-inflammatory effects. Circulation 1995; 92: $1604-1611$
3. Lange LG, Schreiner GF: Immune mechanisms of cardiac disease. $N$ Engl J Med 1994; 330: 1129-1135

4. Matsumori A: Molecular and immune mechanisms in the pathogenesis of cardiomyopathy: Role of viruses, cytokines, and nitric oxide. Jpn Circ J 1997; 61: 275-291

5. McNamara DM, Rosenblum WD, Janosko KM, Trost MK, Villaneuva AJ, Murali S, et al: Intravenous immune globulin in the therapy of myocarditis and acute cardiomyopathy. Circulation 1997; 95: $2476-2478$

6. Shioi T, Matsumori A, Sasayama S: Persistent expression of cytokine in the chronic stage of viral myocarditis in mice. Circulation 1996; 94: 2930-2937

7. Mouthon L, Kaveri SV, Spalter SH, Lacroix-Desmazes S, Lefranc C, Desai R, et al: Mechanisms of action of intravenous immune globulin in immune-mediated diseases. Clin Exp Immunol 1996; 104 (Suppl 1): 3-9

8. Dilated cardiomyopathy and entroviruses (Editorials). Lancet 1990; 336: $971-973$ 